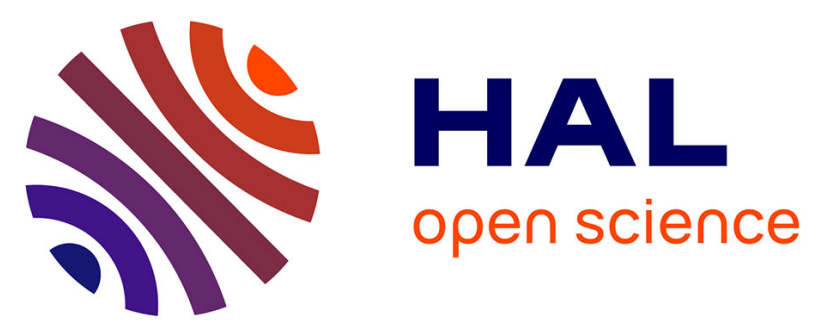

\title{
Quantitative Correlation of Hot Electron Emission to Auger Recombination in the Active Region of c-plane blue III-N LEDs
}

Wan Ying Ho, Yi Chao Chow, Daniel J Myers, Feng Wu, Jacques Peretti, Claude Weisbuch, James S Speck

\section{To cite this version:}

Wan Ying Ho, Yi Chao Chow, Daniel J Myers, Feng Wu, Jacques Peretti, et al.. Quantitative Correlation of Hot Electron Emission to Auger Recombination in the Active Region of c-plane blue III-N LEDs. Applied Physics Letters, 2021. hal-03452309

\section{HAL Id: hal-03452309 https://hal.science/hal-03452309}

Submitted on 26 Nov 2021

HAL is a multi-disciplinary open access archive for the deposit and dissemination of scientific research documents, whether they are published or not. The documents may come from teaching and research institutions in France or abroad, or from public or private research centers.
L'archive ouverte pluridisciplinaire HAL, est destinée au dépôt et à la diffusion de documents scientifiques de niveau recherche, publiés ou non, émanant des établissements d'enseignement et de recherche français ou étrangers, des laboratoires publics ou privés. 


\title{
Quantitative Correlation of Hot Electron Emission to Auger Recombination in the Active Region of $c$-plane blue III-N LEDs
}

\author{
Wan Ying Ho, ${ }^{1}$ Yi Chao Chow, ${ }^{1}$ Daniel J. Myers, ${ }^{1}$ Feng Wu, ${ }^{1}$ Jacques Peretti, ${ }^{2}$ Claude Weisbuch, ${ }^{1,2}$ and James \\ S. Speck ${ }^{1}$ \\ ${ }^{1)}$ Materials Department, University of California, Santa Barbara, California 93106-5050, \\ USA \\ ${ }^{2)}$ Laboratoire de Physique de la Matière Condensée, CNRS, Ecole Polytechnique, IP Paris, 91128 Palaiseau, \\ France
}

(*Electronic mail: wanying_ho@ucsb.edu)

(Dated: 19 July 2021)

Using Electron Emission Spectroscopy (EES), measurement and analysis were conducted on the energy distribution of vacuum emitted electrons from an electrically driven InGaN/GaN commercial blue $c$-plane (peak wavelengths $\lambda \approx$ $465 \mathrm{~nm}$ ) light emitting diode (LED) with $60 \mathrm{~nm}$ of $p$-GaN on top of the active region. The signal-to-noise ratio of semiconductor peaks are improved on the thin p-GaN LED compared to previous published data on thicker $p$-GaN samples and is attributed to reduced loss of electrons en route to emission into vacuum during transit through the $p$-GaN. This further proves that hot electrons are generated in the bulk region and not by light or other hot electron generation mechanism at the surface. Using square root of the light output power (LOP) as a proxy for the active region carrier density, $n$, the hot electron integrated peak intensity is shown to be proportional to $n^{3}$ and thus is directly attributed to a 3-body Auger process. Since there are significant Auger recombination currents even at low injection current densities, it is expected that Auger recombination current will dominate over radiation recombination and Shockley-Read-Hall $(\mathrm{SRH})$ currents at higher current densities. This identifies Auger recombination as the dominant cause of efficiency droop.

As the average luminous efficacy of light emitting diodes (LEDs) has increased over the years, the energy performance of LEDs has surpassed preceding lighting technologies such as incandescent and fluorescent lighting. One way to reduce the cost per lumen hour would be to maximize the wallplug efficiency (WPE), where the internal quantum efficiency (IQE), $\eta_{\text {rad }}$, plays a major role. $\eta_{\text {rad }}$ represents the number of photons created per injected electron-hole $(e-h)$ pair and is known to peak at a low current density $\sim 1-10 \mathrm{~A} \mathrm{~cm}^{-2}$. This reduction in efficiency at higher current densities has been referred to as efficiency droop, or simply droop.

Many mechanisms have been proposed to explain this phenomenon in group III-nitrides LEDs, some of which include carrier escape and overshoot from the active regions, defectassisted tunneling, and carrier delocalization. To date, the dominant mechanism of droop remains a topic of debate. ${ }^{1-4}$ The ABC model is commonly used to describe the shape of the IQE curve in discussions of efficiency droop. A carrier in the quantum well can undergo either: (i) Shockley-ReadHall (SRH) recombination; (ii) radiative recombination; or (iii) band-to-band Auger recombination, which scale as $A n$, $B n^{2}$, and $C n^{3}$ respectively for a given average carrier density, $n$. However, this simplistic model does not account for other forms of non-radiative loss, and there are uncertainties in the fitting of the coefficient $C .5,6$

In 2013, Iveland et al. reported direct measurements of hot electrons from higher side valleys using Electron Emission Spectroscopy (EES) and correlated the appearance and intensity of these hot electrons with efficiency droop. ${ }^{7}$ In EES, the energy distribution of electrons emitted from an electrically driven semiconductor device, such as an LED, can be measured. By realizing negative electron affinity (NEA) on $\mathrm{p}-\mathrm{GaN}$ through the deposition of $\mathrm{Cs}$ or $\mathrm{Cs} / \mathrm{O}$ complexes, elec- trons can be emitted from occupied states near the $\Gamma$ valley bulk position. ${ }^{8}$ While there is little ambiguity in that detection of hot electrons can only be generated by an Auger process, the correlation relied on the extraction of a "supplementary current". The supplementary current was taken as the extra current necessary to realize the measured light output power (LOP) when compared to the "expected" current that would produce the same LOP in the absence of droop. ${ }^{7}$ There is some uncertainty in the procedure defining the supplementary current on which we will improve in the present paper.

In this work, EES was performed on a commercial blue LED with an improved EES device design for enhanced signal-to-noise ratio. ${ }^{9}$ We report on a robust method of analysis that correlates droop with the generation of hot electrons. The blue LED studied in this work was grown using metalorganic chemical vapor deposition (MOCVD). The structure of the epitaxial material is shown in Fig. 1.

The Seoul VioSys LED structure was grown on top of a patterned sapphire substrate and an unintentionally-doped (UID)$\mathrm{GaN}$ buffer layer. The subsequent material consisted of an $n$-type GaN:Si layer, $20 \mathrm{~nm}$ of $\mathrm{InGaN} / \mathrm{GaN}$ superlattice, an 8 period multiple quantum well (QW) (InGaN QW/ AlGaN cap layer/ GaN barrier), a $20 \mathrm{~nm}$ AlGaN:Mg electron blocking layer (EBL), and finally a $40 \mathrm{~nm} \mathrm{GaN}: \mathrm{Mg}\left([\mathrm{Mg}] \sim 2 \times 10^{19}\right.$ $\left.\mathrm{cm}^{-3}\right)$ with $\mathrm{p}++$ contact layer $\left([\mathrm{Mg}]=10^{20} \mathrm{~cm}^{-3}\right)$.

The epitaxial materials were processed into devices suited for EES measurements, cleaned and introduced into the ultrahigh-vacuum (UHV) EES set-up as described elsewhere. ${ }^{7,9}$ The epitaxial materials were contacted with $30 \mathrm{~nm} \mathrm{Pd} / 300$ $\mathrm{nm}$ Au on the $p-\mathrm{GaN}$ in a honeycomb pattern, forming a single EES device of area $0.22 \mathrm{~mm}^{2}$ with an array of 2257 hexagonal open apertures with an apothem of $3.5 \mu \mathrm{m}$ separated by $3 \mu \mathrm{m}$ metal strips. ${ }^{9}$ Negative electron affinity (NEA) was achieved 


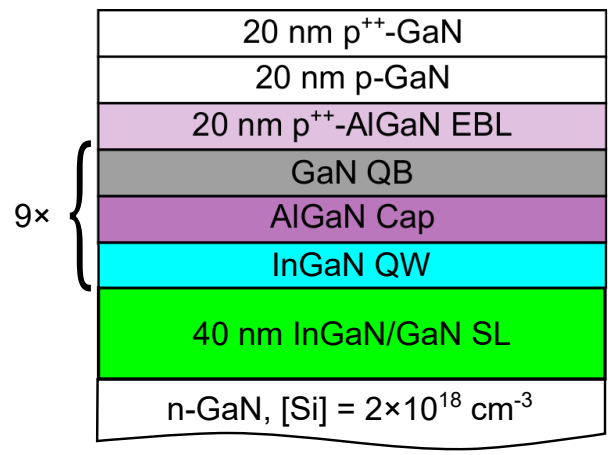

FIG. 1. Schematic of the epi layer stack for the LED discussed in this article, grown by Seoul VioSys.

by depositing a submonolayer of cesium (cesiation) using a SAES cesium source and optimized by monitoring photoexcited electrons emitted from $p$-GaN. ${ }^{7}$ The energy of the electrons was measured with a Comstock AC-901 spherical sector electrostatic analyzer operated in constant pass energy mode with a resolution of $90 \mathrm{meV}$, referenced to the Fermi level of the $p$ contact. ${ }^{7,9}$ As such, with increasing current, there was an increased ohmic voltage drop across the metal-semiconductor interface which shifted the measured energy of electrons emitted from the semiconductor surface to higher values. However, this shift did not affect the Pd and Au photoemission peaks (which are a result of diode light). ${ }^{9,10}$ The LOP of the EES device was measured under continuous wave $(\mathrm{CW})$ mode at room temperature using a photodetector. The $\mathrm{EQE}$ reached its peak value at approximately $10 \mathrm{~A} \mathrm{~cm}^{-2}$. EES was performed with the device biased under pulse mode with a 5\% duty cycle and $1.7 \mu$ s pulses to minimize self-heating, for injection currents ranging from $1 \mathrm{~mA}$ to $100 \mathrm{~mA}$ corresponding to current densities ranging from $0.45 \mathrm{~A} \mathrm{~cm}^{-2}$ to $45 \mathrm{~A} \mathrm{~cm}^{-2}$.

The corresponding energy distribution curves (EDCs) from EES under different currents are shown in Fig. 2(a), where the EDCs for pulsed mode EES were scaled to account for the duty cycle by multiplying with a factor of $20{ }^{7}$ The EDCs show four distinct peaks. Peaks with a semiconductor origin have energies that increase with the applied voltage due to the voltage drop from p-contact to the sample surface. ${ }^{7,9,11}$ The high energy thresholds (HETs) of the peaks can be extracted from the negative parts of the derivative of the EDCs, and equal to the extrapolated x-intercepts of the high energy slopes. ${ }^{7,9,11}$ The extrapolated HETs at the expected turn-on voltage of $2.67 \mathrm{~V}$ should correspond to the bulk valley minimum as shown in Fig. 2(b). ${ }^{7,9,11}$ Based on the extrapolated values, we assign the measured peaks in increasing energies in the following order: Au PE and Pd PE (both peaks are due to diode light), $\Gamma$ and first side valley at $\sim 0.9 \mathrm{eV}$ higher energy than $\Gamma{ }^{9-14}$ A low energy shoulder appears on $\Gamma$ valley at high current density, which may be ascribed to thermalization or subbandgap PE through Franz-Keldysh absorption in the band-bending region. ${ }^{7}$ The semiconductor related peaks are one or two orders of magnitude larger than our previous works which employed thicker $p$-regions, showing significant
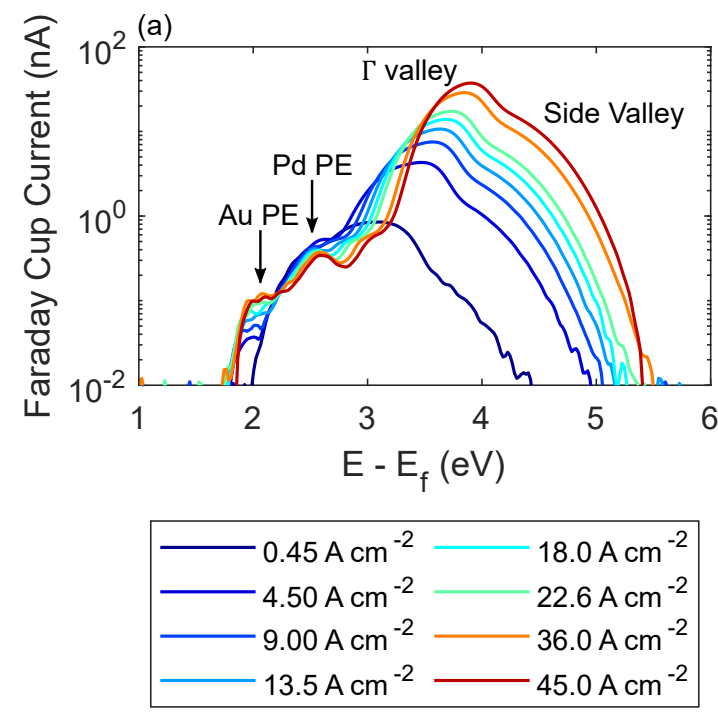

(b)
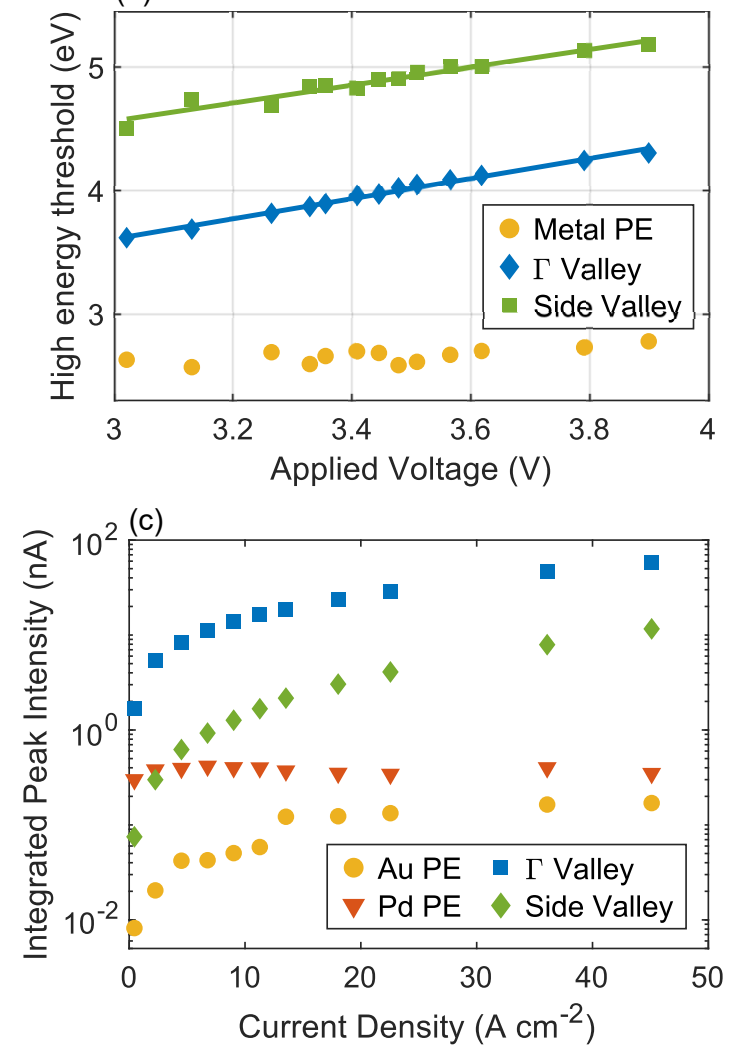

FIG. 2. The EDCs, peak high energy thresholds, and integrated peak intensities are plotted in (a) - (c) respectively. For all peaks, except $\mathrm{Pd} P E$, the integrated intensities increase with current density. The Pd PE varied very slightly with increasing current densities but the differences are within EDC background noise levels. 
improvement in signal-to-noise ratio. ${ }^{7,9-11}$ This further implied that hot electrons are indeed generated in the bulk region and not by light or other hot electron generation mechanism at the surface.

The magnitude of $\Gamma$ valley is higher than that of the side valley, unlike previous reported EES spectra of commercial LEDs with much thicker $p$-GaN, ${ }^{7,9-11}$ but more similar to Ref. 15 where the sample had $85 \mathrm{~nm} p$-GaN. ${ }^{15}$ This is notable as it suggests that with thinner $p$-GaN more $\Gamma$ valley electrons can reach the sample surface to be emitted. In fact, the side valley mainly depopulates by scattering into the $\Gamma$ valley since the side valley electrons have very few recombination pathways due to weaker radiative recombination or SRH recombination because of the larger energy difference and limited k-extension of defects. ${ }^{13}$ In contrast, electrons in $\Gamma$ valley, whether scattered from the side valley or overflowed from the active region, may recombine radiatively or non-radiatively (likely the dominant mechanism) within a diffusion length. One can then assume that the side valley depopulates less during the transit through the $p$-region to the surface than the $\Gamma$ valley. ${ }^{14}$ Therefore, the ratio of the $\Gamma$ valley electron population to the side valley electron population may increase when decreasing the $p$-GaN layer thickness.

Analytic fits to the EES spectra were performed by superposing four exponentially modified Gaussians to the four EES peaks, respectively. ${ }^{11}$ The dependence of the integrated intensities (given as a current) of the four peaks on the LED current density are shown in Fig. 2(c). As expected, the integrated peak intensities of Au PE, $\Gamma$ valley and side valley increases with increasing current density. The Pd PE peak intensity increased initially but remained almost constant at higher current densities, a saturation that was observed in previous work and might be due to the overlap with the peak assigned to the subbandgap PE peak generated in the BBR electric field. ${ }^{9}$

The Au PE intensities acts like an in-situ photometer, ${ }^{11}$ varying linearly with the LOP of the device. Since every electron in the Au PE peak must correspond to one photon emitted by the LED, we expect both the LOP and Au PE peak intensity to vary as radiative recombination rate, hence vary as $n^{2}$. Similarly, if hot electrons in the side valley originate from $e e h$ recombination, we expect the peak intensity to vary as $n^{3}$. Hence the side valley peak intensities should vary linearly as $\mathrm{Au}$ PE peak intensity to the power of 3/2. Since the Au PE peaks had been partially masked by Pd PE and semiconductor peaks and hence is subject to fitting errors, a similar analysis was repeated using LOP to the power of $3 / 2$ and comparing to the side valley electron intensity. A good linear fit was obtained with a Pearson's coefficient of 0.992 as shown in Fig. 3(a). The produced intercept of $0.02 \mathrm{nA}$ is of the order of the background noise in the EDCs. From the good fits, we conclude that the side valley electrons originate from 3-body eeh Auger events. Interestingly, the intensity of $\Gamma$ valley electrons demonstrated a partial cubic dependence of $\mathrm{n}$ at higher current densities as shown in Fig. 3(b). A least square curve fit is attempted instead where the fits are bounded to only return positive coefficients for $n^{2}$ and $n^{3}$ terms to keep the analysis physically viable. No $n$ term is included as Shockley-ReadHall recombination is incapable of generating hot electrons.
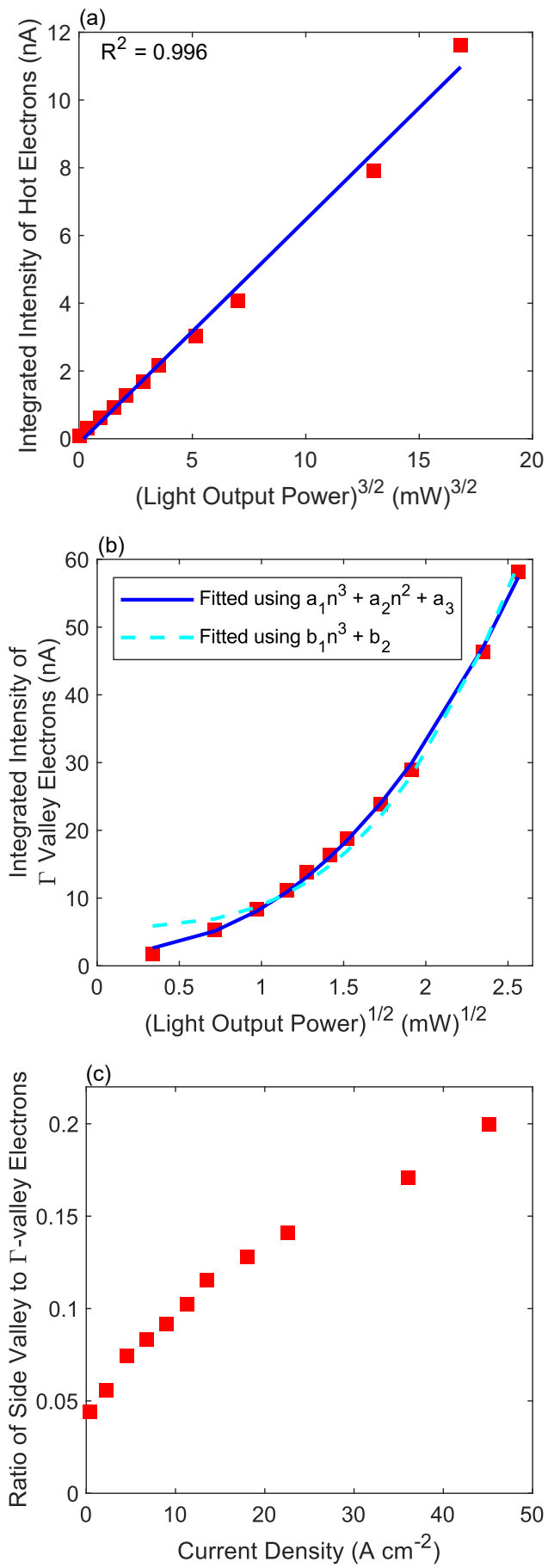

FIG. 3. In (a), by comparing the cube of the square root of LOP, which is proportional to $n^{3}$ with the side valley peak intensity, a good linear correlation is obtained. In (b), it is found that the $\Gamma$ valley peak intensities demonstrated a similar cubic dependence of $n$. A quadratic term is required for improved fit. In (c), the ratio of hot electrons in first side valley to electrons in $\Gamma$ valley is found to increase with increasing current density, ruling out escape and overshoot as cause of droop. 
The fit was improved significantly when a quadratic term is included into the fit, with the norm of residuals decreasing from 33.7 to 4.0 , and intercept tending towards zero from 5.75 to $1.97 \mathrm{nA}$. While introducing a lower power term is expected to improve fits at lower current densities, the fit is improved even past the peak EQE in the droop regime. This $n^{2}$ dependence of hot electrons may be attributed to trap-assisted Auger recombination (TAAR). ${ }^{11,15,16}$

At the same time, the LED has an EBL in its structure, which is expected to mitigate both escape and overshoot of electrons from the active region. ${ }^{1}$ Simulations also showed that leakage currents are small compared to Auger currents. ${ }^{17}$ Should either phenomenon be present, we should detect these events as presence of $\Gamma$ valley electrons in the EDCs as they will not have enough energy to reach the side valley. ${ }^{10}$ If either were the dominant cause of droop, we would expect the peak intensities of $\Gamma$ valley to increase relative to the side valley peak with increasing current density. As shown in Fig. 3(c), this is not the case, strongly indicating that the rate of increase of escape and overshoot currents with increasing current density must be low compared to that of Auger currents and should not cause droop. Combining this knowledge with the carrier density dependence of $\Gamma$ valley electrons, it is possible that the source of detected $\Gamma$ valley electrons is either by scattering of Auger electrons from the side valley, and/or by 3-body eeh or 2-body TAAR events. ${ }^{11,15,16}$ Therefore the integrated intensity of $\Gamma$ valley electrons is also a good proxy for hot carriers. Given the $n^{3}$ dependence of 3-body Auger recombination, it is expected that Auger recombination current will dominate rapidly over radiative recombination current with increasing injected carrier density. The presence of detected side valley electrons even at very low current densities implies that the Auger recombination current is significant in magnitude and should lead to droop. ${ }^{7,12}$ Thus, we conclude that droop in this LED must be hence dominantly caused by Auger recombination.

In conclusion, we have measured hot electrons from a higher energy side valley in addition to those from the conduction band minimum with vastly improved semiconductor peak signal-to-noise ratio attributed to reduced electron loss due to use of thinner $p$-GaN. By integrating peaks and investigating the correlation of their intensities with the LOP, we have proven that hot electrons originate dominantly from a 3-body event. This further confirms that efficiency droop is dominantly caused by Auger recombination.

\section{SUPPLEMENTARY MATERIAL}

See supplementary material for analysis performed on other commercial blue $c$-plane LEDs with the same method.

\section{ACKNOWLEDGMENTS}

The samples were kindly provided by Seoul VioSys and Cree, Inc. Support at UCSB was provided by the Univer- sity of California, Santa Barbara (UCSB) - Collaborative Research of Engineering, Science and Technology (CREST) program; the Solid State Lighting and Energy Electronics Center (SSLEEC); the Simons Foundation (Grant Nos. 601952 and 601954 for JSS and CW, respectively); and the National Science Foundation (NSF) RAISE program (Grant No. DMS-1839077) and Sandia National Laboratory (Award No. 2150283). A portion of this work was performed at the UCSB Nanofabrication facility. We are thankful to Kai Shek Qwah for his contribution.

\section{DATA AVAILABILITY STATEMENT}

The data that support the findings of this study are available from the corresponding author upon reasonable request.

${ }^{1}$ V. Avrutin, S. d. A. Hafiz, F. Zhang, U. Özgür, H. Morkoç, and A. Matulionis, Journal of Vacuum Science \& Technology A 31, 050809 (2013), https://doi.org/10.1116/1.4810789.

${ }^{2}$ G. Verzellesi, D. Saguatti, M. Meneghini, F. Bertazzi, M. Goano, G. Meneghesso, and E. Zanoni, Journal of Applied Physics 114, 071101 (2013), https://doi.org/10.1063/1.4816434.

${ }^{3}$ J. Cho, E. F. Schubert, and J. K. Kim, Laser \& Photonics Reviews 7, 408 (2013), https://onlinelibrary.wiley.com/doi/pdf/10.1002/lpor.201200025.

${ }^{4}$ J.-I. Shim, III-Nitride Based Light Emitting Diodes and Applications, edited by T.-Y. Seong, J. Han, H. Amano, and H. Morkoç (Springer, Dordrecht, 2013).

${ }^{5}$ H.-Y. Ryu, H.-S. Kim, and J.-I. Shim, Applied Physics Letters 95, 081114 (2009), https://doi.org/10.1063/1.3216578.

${ }^{6}$ C. Weisbuch, M. Piccardo, L. Martinelli, J. Iveland, J. Peretti, and J. S. Speck, physica status solidi (a) 212, 899 (2015), https://onlinelibrary.wiley.com/doi/pdf/10.1002/pssa.201431868.

${ }^{7}$ J. Iveland, L. Martinelli, J. Peretti, J. S. Speck, and C. Weisbuch, Phys. Rev. Lett. 110, 177406 (2013).

${ }^{8}$ O. E. Tereshchenko, G. É. Shaibler, A. S. Yaroshevich, S. V. Shevelev, A. S. Terekhov, V. V. Lundin, E. E. Zavarin, and A. I. Besyul'kin, Physics of the Solid State 46, 1949 (2004).

${ }^{9}$ D. J. Myers, K. Gelžinytė, W. Y. Ho, J. Iveland, L. Martinelli, J. Peretti, C. Weisbuch, and J. S. Speck, Journal of Applied Physics 124, 055703 (2018), https://doi.org/10.1063/1.5030208.

${ }^{10}$ J. Iveland, M. Piccardo, L. Martinelli, J. Peretti, J. W. Choi, N. Young, S. Nakamura, J. S. Speck, and C. Weisbuch, Applied Physics Letters 105, 052103 (2014), https://doi.org/10.1063/1.4892473.

${ }^{11}$ D. J. Myers, K. Gelžinytė, A. I. Alhassan, L. Martinelli, J. Peretti, S. Nakamura, C. Weisbuch, and J. S. Speck, Phys. Rev. B 100, 125303 (2019).

${ }^{12}$ M. Piccardo, L. Martinelli, J. Iveland, N. Young, S. P. DenBaars, S. Nakamura, J. S. Speck, C. Weisbuch, and J. Peretti, Phys. Rev. B 89, 235124 (2014).

${ }^{13}$ S. Wu, P. Geiser, J. Jun, J. Karpinski, D. Wang, and R. Sobolewski, Journal of Applied Physics 101, 043701 (2007), https://doi.org/10.1063/1.2496399.

${ }^{14}$ S. Marcinkevičius, T. K. Uždavinys, H. M. Foronda, D. A. Cohen, C. Weisbuch, and J. S. Speck, Phys. Rev. B 94, 235205 (2016).

${ }^{15}$ D. J. Myers, A. C. Espenlaub, K. Gelzinyte, E. C. Young, L. Martinelli, J. Peretti, C. Weisbuch, and J. S. Speck, Applied Physics Letters 116, 091102 (2020), https://doi.org/10.1063/1.5125605.

${ }^{16}$ A. C. Espenlaub, D. J. Myers, E. C. Young, S. Marcinkevičius, C. Weisbuch, and J. S. Speck, Journal of Applied Physics 126, 184502 (2019), https://doi.org/10.1063/1.5096773.

${ }^{17}$ C.-K. Li, M. Piccardo, L.-S. Lu, S. Mayboroda, L. Martinelli, J. Peretti, J. S. Speck, C. Weisbuch, M. Filoche, and Y.-R. Wu, Phys. Rev. B 95, 144206 (2017). 